\title{
Satisfacción y compromiso laboral del personal de salud de la microred de San Juan Bautista - Ayacucho, 2014
}

\author{
Commitment and job \\ satisfaction of health \\ personnel of microred \\ San Juan Bautista- \\ Ayacucho, 2014
}

\section{Nelly Felipa Ledesma Zerpa ${ }^{1}$}

1. Maestra en Gerencia de Servicios de Salud, Past Gerente de la Micro Red de Salud de San Juan Bautista, Obstetra Esp. del Centro de Salud de San Juan Bautista, Ayacucho-Perú.

\section{ABSTRACT}

INTRODUCTION: The commitment and job satisfaction study of health Micro Red San Juan Bautista was structured based on the premise that every organization exists only when people come together to cooperate with each other and achieve common institutional goals, where as staff health you can not develop quality job if you are not satisfied for their job performance, so the targets were set OBJETIVE: To determine the relation ship between satisfaction and work commitment of health personnel in Micro Red San Juan Bautista, Ayacucho. MATERIALS AND METHODS research is application, quantitative design, it is a prospective, descriptive correlational cross-sectional. Study Area, Health Facilities Micro Red of San Juan Bautista, sample, consisting of 102 workers (professionals and not professionals) Micro Red of San Juan Bautista; type of sampling probability. Instruments were: Satisfaction survey of Health Personnel Scale and Commitment. RESULTS; $71.6 \%$ of the staff of the Micro Red San Juan Bautista has an average organizational commitment and $44.1 \%$ of them have a personnel and according to the satisfaction chi square test, there is significant relation ship between both variables accepting the research hypothesis, meaning that to the extent that personnel are more satisfied have greater involvement in the work performed.

KEYWORDS: Job satisfaction, job commitment.
PALABRAS CLAVE: Satisfacción laboral, Compromiso laboral. 


\section{INTRODUCCIÓN}

El personal en los servicios de salud representa al capital humano de toda organización, es el recurso más valioso en la institución pública o privada, por ello se debe de contar con políticas y programas conducentes al mejoramiento de la calidad de vida en el entorno laboral ${ }^{1}$. La satisfacción laboral es un indicador de calidad del servicio y desarrollo organizacional ${ }^{2}$. El compromiso laboral incluye la aceptación de las metas de la organización, la disposición a realizar esfuerzos en pro de ésta ${ }^{5}$. En los resultados de la Red Huamanga acerca de la evaluación de las actividades de Acuerdo de Gestión 2011, se escucharon expresiones que de algún modo denotaron insatisfacción laboral, donde el personal de los Establecimientos de salud se quejaba por los menores logros obtenidos en la falta de cobertura de metas en comparación con otros Establecimientos. Atribuyendo a la falta de un buen trato entre compañeros, condiciones de trabajo inadecuados, escasos materiales e insumos, entre otros. Basado en las premisas mencionadas surge la necesidad de estudiar ¿Cuál es la relación entre la satisfacción y compromiso laboral del personal de salud de la Micro Red de San Juan Bautista- Ayacucho, 2014? Objetivo General: Determinar la relación entre la satisfacción y el compromiso laboral del personal de salud en la Micro Red de San Juan Bautista-Ayacucho 2014. Objetivos Específicos: Identificar la satisfacción del personal de salud de la Micro Red de San Juan Bautista. Establecer el compromiso laboral del personal de salud de la Micro Red de San Juan Bautista en las dimensiones de, participación, identificación, sentimiento de pertenencia y lealtad. Relacionar la satisfacción con el compromiso laboral del personal de salud. La Hipótesis de investigación Hi la satisfacción tiene relación directa con el compromiso laboral del personal de salud en la Micro Red de San Juan BautistaAyacucho 2014.

\section{MATERIAL Y MÉTODOS}

El enfoque de investigación fue cuantitativo, el tipo de investigación aplicativo de nivel correlacional de diseño no experimental, transversal relacional. Área de investigación: Establecimientos de salud de la Micro Red de San Juan Bautista (Centro de salud de San Juan B, PS de Miraflores, PS de los Olivos, PS de Ñahuinpuquio, PS de Manallasacc, PS de Sachabamba). Población: Estuvo constituida por 174 trabajadores (profesionales y no profesionales). Para determinar la muestra se aplicó la fórmula para población finita. La muestra estuvo constituida por 102 trabajadores que cumplieron con los criterios de inclusión y exclusión.

\begin{tabular}{|ccc|}
\hline \multicolumn{1}{|c|}{ CRITERIOS } & INCLUSIÓN & EXCLUSIÓN \\
\hline Condición laboral & Nombrada, contratada & SERUMS \\
Tiempo de servicio & $>1$ año & $<0=1$ año \\
\hline
\end{tabular}

Para determinar la muestra proporcional en cada establecimiento de salud se usó la fórmula para submuestras por estratos

\begin{tabular}{|ccc|}
\hline Establecimientos & Nh & nh \\
\hline CS de San Juan Bautista & 110 & 64 \\
PS de Miraflores & 21 & 12 \\
PS de Los Olivos & 20 & 12 \\
PS de Ñahuinpuquio & 15 & 09 \\
PS de Sachabamba & 03 & 02 \\
PS de Manallasacc & 05 & 03 \\
Total & 174 & 102 \\
\hline
\end{tabular}

Para determinar la satisfacción del personal se utilizó la encuesta elaborada y validada por el MINSA 2002. Aspectos que considera la encuesta: Trabajo actual, trabajo en general, interacción con el jefe inmediato, oportunidades de progreso, remuneración e incentivos, interrelación con sus compañeros de trabajo, ambiente de trabajo. El instrumento es una encuesta de 22 ítems de una escala de Likert de 5 opciones asignándole a cada uno un valor en puntos. Para determinar el compromiso laboral se utilizó la encuesta de escala de compromiso elaborado por Grajales y Valderrama 2005 conformado por 21 ítems distribuido en 4 dimensiones y son: participación, identificación, pertenencia, lealtad. Cada una de las declaraciones tiene 5 posibles respuestas según la escala con un puntaje mínimo de 21 y máximo de 105 puntos. La confiabilidad estuvo determinada por cada uno de los autores que elaboraron los instrumentos. Procedimientos: Mediante el Dr. De la Escuela de Posgrado se solicitó la autorización para la realización del trabajo a la Gerencia de la Micro Red de San Juan Bautista. Los instrumentos fueron aplicados en los Establecimientos de la Micro Red. Los datos fueron procesados empleando el paquete estadístico SPSS versión 19.0 (español). Para el análisis se recurrió a la estadística inferencial. Se aplicó la confidencialidad de las respuestas.

\section{RESULTADOS}




\section{TABLA No 1}

Satisfacción laboral del personal de salud en la Micro Red de San Juan B. Ayacucho 2014

\begin{tabular}{|lcc|}
\hline Satisfacción del personal & $\mathbf{N}^{\circ}$ & $\%$ \\
\hline satisfacción & 71 & 69.6 \\
\hline insatisfacción & 31 & 30.4 \\
\hline Total & 102 & 100 \\
\hline
\end{tabular}

En el cuadro referente a la satisfacción laboral del personal de salud se observa que del total de población en estudio 102 (100\%), el 69.6\% de población tiene satisfacción laboral y $30.4 \%$ tiene insatisfacción laboral en la Micro Red.

\begin{tabular}{|c|c|c|c|c|c|c|}
\hline \multirow[t]{3}{*}{ categorías de satisfacción } & \multicolumn{4}{|c|}{ Satisfacción del personal } & \multirow{2}{*}{\multicolumn{2}{|c|}{ Total }} \\
\hline & \multicolumn{2}{|c|}{ Satisfacción } & \multicolumn{2}{|c|}{ insatisfacción } & & \\
\hline & $\mathbf{N}^{\circ}$ & $\%$ & $\mathbf{N}^{\circ}$ & $\%$ & $\mathbf{N}^{\circ}$ & $\%$ \\
\hline Trabajo actual & 80 & 78.4 & 22 & 21.6 & 102 & 100.0 \\
\hline Trabajo en general & 81 & 79.4 & 21 & 20.6 & 102 & 100.0 \\
\hline $\begin{array}{l}\text { Interacción con el jefe } \\
\text { inmediato }\end{array}$ & 52 & 51.0 & 50 & 49.0 & 102 & 100.0 \\
\hline Oportunidades de progreso & 56 & 54.9 & 46 & 45.1 & 102 & 100.0 \\
\hline $\begin{array}{l}\text { Remuneraciones e } \\
\text { incentivos }\end{array}$ & 52 & 51.0 & 50 & 49.0 & 102 & 100.0 \\
\hline $\begin{array}{l}\text { Interrelación con los } \\
\text { compañeros de trabajo }\end{array}$ & 62 & 60.8 & 40 & 39.2 & 102 & 100.0 \\
\hline Ambiente de trabajo & 75 & 73.5 & 27 & 26.5 & 102 & 100.0 \\
\hline
\end{tabular}

En el cuadro referente a la satisfacción del personal según categorías de satisfacción, del total de población en estudio 102 (100\%) el mayor porcentaje de población se encuentran satisfechos en las diferentes categorías, de ellos el $78.4 \%$ se encuentran satisfechos en la categoría de trabajo actual; $79.4 \%$ se encuentran satisfechos en la categoría trabajo en general, el $73.5 \%$ se encuentran satisfechos en la categoría ambiente de trabajo, así mismo el $60.8 \%$ se encuentran satisfechos en la categoría interrelación con los compañeros de trabajo.

\section{TABLA No 3}

Compromiso organizacional del personal de salud en la Micro Red de San Juan Bautista -Ayacucho 2014

\begin{tabular}{|ccc|}
\hline $\begin{array}{c}\text { Compromiso } \\
\text { organizacional }\end{array}$ & $\mathbf{N}^{\circ}$ & $\%$ \\
\hline Alta & 27 & 26.5 \\
Media & 73 & 71.6 \\
Baja & 2 & 2.0 \\
\hline Total & 102 & 100.0 \\
\hline
\end{tabular}

En el cuadro referente al compromiso organizacional del personal de salud en la Micro Red se observa que del total de población en estudio 102 (100\%) el 71.6\% de población tiene un compromiso organizacional medio, $26.5 \%$ alto y $2.0 \%$ un compromiso bajo.

\section{TABLA № 4}

Categorías según compromiso organizacional del personal de salud en la Micro Red de San Juan Bautista Ayacucho 2014

\begin{tabular}{|c|c|c|c|c|c|c|c|c|}
\hline \multirow[t]{3}{*}{ categorías } & \multicolumn{8}{|c|}{ Compromiso organizacional } \\
\hline & \multicolumn{2}{|c|}{ Alta } & \multicolumn{2}{|c|}{ Media } & \multicolumn{2}{|c|}{ Baja } & \multicolumn{2}{|c|}{ total } \\
\hline & $\mathbf{N}^{\circ}$ & $\%$ & $\mathbf{N}^{\circ}$ & $\%$ & $\mathbf{N}^{\circ}$ & $\%$ & $\mathbf{N}^{\circ}$ & $\%$ \\
\hline Participación & 41 & 40.2 & 53 & 52.0 & 8 & 7.8 & 102 & 100.0 \\
\hline Identificación & 80 & 78.4 & 21 & 20.6 & 1 & 1.0 & 102 & 100.0 \\
\hline Pertenencia & 10 & 9.8 & 40 & 39.2 & 52 & 51.0 & 102 & 100.0 \\
\hline Lealtad & 25 & 24.5 & 59 & 57.8 & 18 & 17.6 & 102 & 100.0 \\
\hline
\end{tabular}

En el cuadro referente al compromiso organizacional del personal según categorías de compromiso organizacional, del total de población en estudio 102 (100\%), el $52.0 \%$ tiene el compromiso organizacional medio y el $40.2 \%$ alto en la categoría de participación; del mismo modo se observa que en la categoría de identificación, el $78.4 \%$ tiene el compromiso organizacional alto, $20.6 \%$ medio; en la categoría pertenencia el $51.0 \%$ tienen el compromiso organizacional bajo, $39.2 \%$ medio y en la categoría lealtad el $57.8 \%$ tienen el compromiso organizacional medio, $24.5 \%$ alto. 


\section{TABLA No 5}

Satisfacción y grado de compromiso laboral según puesto laboral del personal de salud en la Micro Red de San Juan Bautista-Ayacucho 2014

\begin{tabular}{|c|c|c|c|c|c|c|c|c|c|c|c|c|c|c|}
\hline \multirow{3}{*}{$\begin{array}{l}\text { Puesto } \\
\text { laboral }\end{array}$} & \multicolumn{4}{|c|}{ Satisfacción del personal } & \multirow{2}{*}{\multicolumn{2}{|c|}{ Total }} & \multicolumn{4}{|c|}{ Compromiso laboral } & & & \multicolumn{2}{|c|}{ Total } \\
\hline & \multicolumn{2}{|c|}{ satisfación } & \multicolumn{2}{|c|}{ insatisfaccio } & & & \multicolumn{2}{|c|}{ Alta } & \multicolumn{2}{|c|}{ Media } & \multicolumn{2}{|c|}{ Baja } & \multirow[b]{2}{*}{$\mathbf{N}^{\circ}$} & \multirow[b]{2}{*}{$\%$} \\
\hline & $\mathbf{N}^{\circ}$ & $\%$ & $\mathbf{N}^{\circ}$ & $\%$ & $\mathbf{N}^{\circ}$ & $\%$ & $\mathbf{N}^{\circ}$ & $\%$ & $\mathbf{N}^{\circ}$ & $\%$ & $\mathbf{N}^{\circ}$ & $\%$ & & \\
\hline Gerente & 1 & 1.0 & 0 & 0.0 & 1 & 1.0 & 0 & 0.0 & 1 & 1.0 & 0 & 0.0 & 1 & 1.0 \\
\hline Jefe de dpto. & 10 & 9.8 & 3 & 2.9 & 13 & 12.7 & 3 & 2.9 & 10 & 9.8 & 0 & 0.0 & 13 & 12.7 \\
\hline Personal as. & 50 & 49.0 & 23 & 22.5 & 73 & 71.6 & 22 & 21.6 & 50 & 49.0 & 1 & 1.0 & 73 & 71.6 \\
\hline Personal ad. & 7 & 6.9 & 4 & 3.9 & 11 & 10.8 & 1 & 1.0 & 10 & 9.8 & 0 & 0.0 & 11 & 10.8 \\
\hline Personal ser. & 1 & 1.0 & 1 & 1.0 & 2 & 2.0 & 1 & 1.0 & 0 & 0.0 & 1 & 1.0 & 2 & 2.0 \\
\hline Otro & 2 & 2.0 & 0 & 0.0 & 2 & 2.0 & 0 & 0.0 & 2 & 2.0 & 0 & 0.0 & 2 & 2.0 \\
\hline Total & 71 & 69.6 & 31 & 30.4 & 102 & 100.0 & 27 & 26.5 & 73 & 71.6 & 2 & 2.0 & 102 & 100.0 \\
\hline
\end{tabular}

$\begin{array}{lllll}x^{2}(c)=2.23 & x^{2}(t)=4.35 & G l=5 & \text { N.C }=0.5 & p<0,05 \\ x^{2}(c)=29.53 & x^{2}(t)=9.34 & G l=10 & \text { N.C }=0.5 & p>0,05\end{array}$

En el cuadro referente a la satisfacción y el grado de compromiso laboral según el puesto laboral del personal de salud de la MR de San Juan Bautista, se observa que del total de población en estudio 102 $(100 \%)$, el $71.6 \%$ es personal asistencial de ellos el $49.0 \%$ tienen satisfacción laboral y el $21.6 \%$ compromiso laboral alto; del mismo modo el $12.7 \%$ ejerce como jefe de departamento, de ellos $9.8 \%$ tiene satisfacción y compromiso laboral medio; asimismo el $10.8 \%$ labora como personal administrativo, de ellos $6.9 \%$ tiene satisfacción laboral y $9.8 \%$ tiene compromiso laboral medio, se puede observar que el $2.0 \%$ ejerce como personal de servicio y otros respectivamente; de ellos 1.0\% tiene insatisfacción y bajo compromiso laboral. De acuerdo a la prueba de chi cuadrada en lo que respecta a la satisfacción personal se afirma que no existe relación significativa entre la satisfacción personal y el puesto laboral del personal de salud. En lo que respecta al compromiso laboral se afirma que existe relación significativa entre el compromiso laboral y el puesto laboral del personal de salud en la Micro Red de San Juan Bautista.

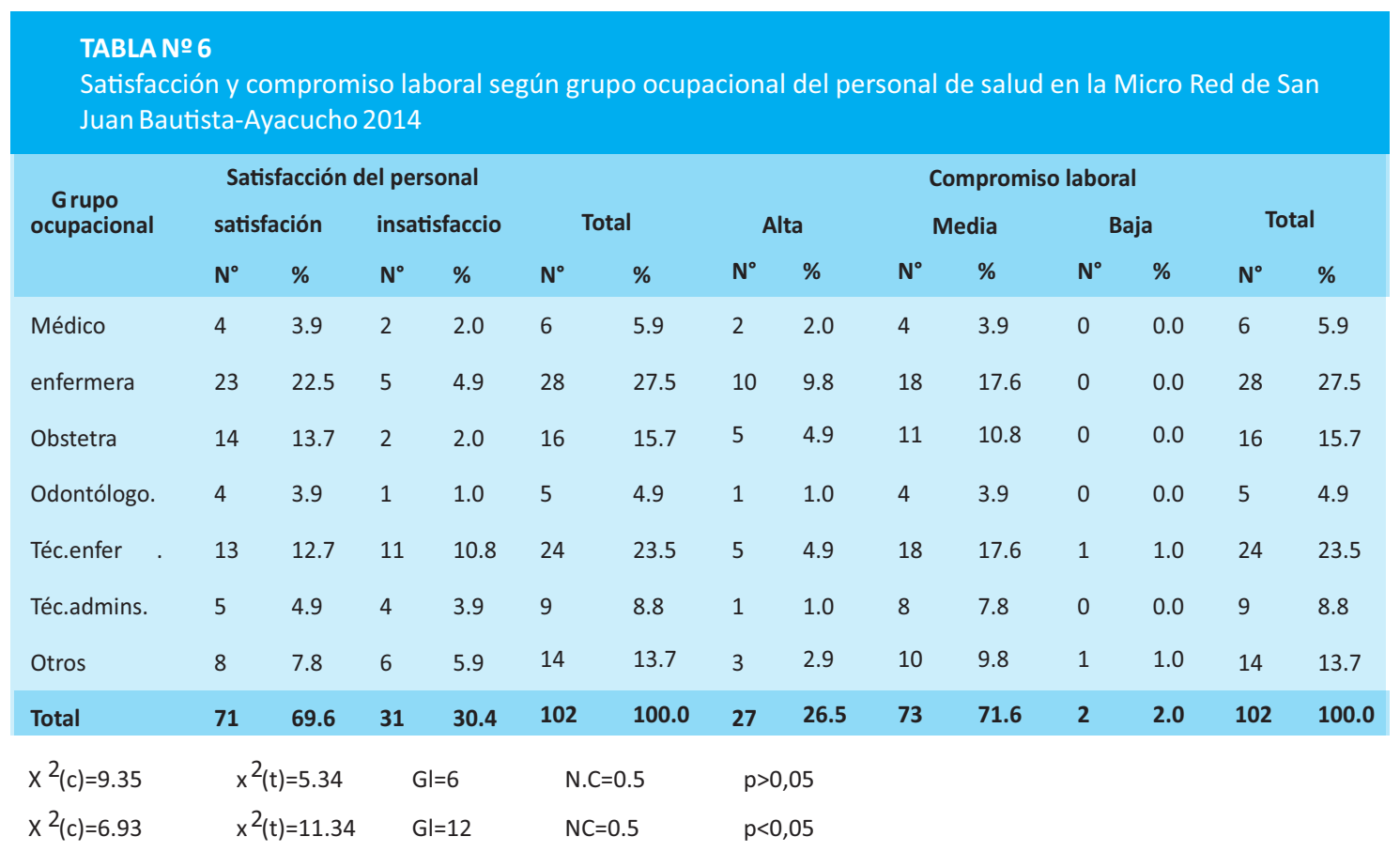


En el cuadro referente a la satisfacción y compromiso laboral según grupo ocupacional del personal de salud de la MR de San Juan Bautista se observa que del total de población en estudio 102 (100\%) el 27.5\% pertenece al grupo ocupacional de licenciados en enfermería de ellos el $22.5 \%$ tiene satisfacción laboral y $17.6 \%$ tiene compromiso laboral medio, asimismo el $23.5 \%$ pertenece al grupo de técnicos en enfermería de ellos $12.7 \%$ tiene satisfacción laboral y $17.6 \%$ tiene compromiso laboral medio; del mismo modo el $15.7 \%$ pertenece al grupo de obstetras, de ellos $13.7 \%$ tiene satisfacción laboral y $10.8 \%$ tiene compromiso laboral medio, también se puede observar que el $8.8 \%$ pertenece al grupo de técnicos administrativos de ellos $4.9 \%$ tiene satisfacción laboral y $7.8 \%$ tiene compromiso laboral medio. De acuerdo a la prueba de chi cuadrada en lo que respecta a la satisfacción personal se afirma que existe relación significativa entre la satisfacción personal y grupo ocupacional del personal de salud en la MR de San Juan Bautista. En lo que respecta al compromiso laboral se afirma que no existe relación significativa entre el compromiso laboral y grupo ocupacional del personal de salud.

\begin{tabular}{|c|c|c|c|c|c|c|c|c|c|c|c|c|c|c|}
\hline \multirow{2}{*}{$\begin{array}{l}\text { Condición } \\
\text { laboral }\end{array}$} & \multicolumn{4}{|c|}{ Satisfacción del personal } & \multicolumn{10}{|c|}{ Compromiso laboral } \\
\hline & $\mathbf{N}^{\circ}$ & $\%$ & $\mathbf{N}^{\circ}$ & $\%$ & $\mathbf{N}^{\circ}$ & $\%$ & $\mathbf{N}^{\circ}$ & $\%$ & $\mathbf{N}^{\circ}$ & $\%$ & $\mathbf{N}^{\circ}$ & $\%$ & $\mathbf{N}^{\circ}$ & $\%$ \\
\hline Nombrado & 47 & 46.1 & 27 & 26.5 & 74 & 72.5 & 18 & 17.6 & 54 & 52.9 & 2 & 2.0 & 74 & 72.5 \\
\hline Contratad & 14 & 13.7 & 3 & 2.9 & 17 & 16.7 & 5 & 4.9 & 12 & 11.8 & 0 & 0.0 & 17 & 16.7 \\
\hline Otros & 10 & 9.8 & 1 & 1.0 & 11 & 10.8 & 4 & 3.9 & 7 & 6.9 & 0 & 0.0 & 11 & 10.8 \\
\hline Total & 71 & 69.6 & 31 & 30.4 & 102 & 100.0 & 27 & 26.5 & 73 & 71.6 & 2 & 2.0 & 102 & 100.0 \\
\hline$x^{2}(c)=5.0$ & & $=1.38$ & & $\mathrm{Gl}=2$ & N.C $=$ & & & & & & & & & \\
\hline$x^{2}(c)=1.47$ & & $t)=3.35$ & & $\mathrm{Gl}=4$ & N.C $=$ & & $p<0$ & & & & & & & \\
\hline
\end{tabular}

En el cuadro referente a la satisfacción y compromiso laboral según condición laboral del personal de salud de la Micro Red de SJB se observa que del total de población en estudio 102 (100\%) el 72.5\% tienen la condición laboral de nombrados, de ellos el $46.1 \%$ tiene satisfacción laboral y $52.9 \%$ tiene compromiso laboral medio, asimismo el $16.7 \%$ tiene la condición laboral de contratado, de ellos $13.7 \%$ tiene satisfacción laboral del mismo modo10.8\% tiene otro tipo de condición laboral, de ellos $9.8 \%$ tiene satisfacción laboral y $6.9 \%$ tiene un compromiso laboral medio. De acuerdo a la prueba de chi cuadrada en lo que respecta a la satisfacción personal se afirma que existe relación significativa entre la satisfacción del personal y la condición laboral del personal de salud. En lo que respecta al compromiso laboral se afirma que no existe relación significativa entre el compromiso laboral y la condición laboral del personal de salud de la MicroRed de San Juan Bautista.

\begin{tabular}{|c|c|c|c|c|c|c|c|c|c|c|c|c|c|c|}
\hline \multirow{3}{*}{$\begin{array}{l}\text { Tiempo de } \\
\text { servicio }\end{array}$} & \multicolumn{4}{|c|}{ Satisfacción del personal } & \multicolumn{10}{|c|}{ Compromiso laboral } \\
\hline & \multicolumn{2}{|c|}{ satisfación } & \multicolumn{2}{|c|}{ insatisfaccio } & \multicolumn{2}{|c|}{ Total } & \multicolumn{2}{|c|}{ Alta } & \multicolumn{2}{|c|}{ Media } & \multicolumn{2}{|c|}{ Baja } & \multicolumn{2}{|c|}{ Total } \\
\hline & $\mathbf{N}^{\circ}$ & $\%$ & $\mathbf{N}^{\circ}$ & $\%$ & $\mathbf{N}^{\circ}$ & $\%$ & $\mathbf{N}^{\circ}$ & $\%$ & $\mathbf{N}^{\circ}$ & $\%$ & $\mathbf{N}^{\circ}$ & $\%$ & $\mathbf{N}^{\circ}$ & $\%$ \\
\hline $\begin{array}{l}\text { Menos de un } \\
\text { año. }\end{array}$ & 1 & 1.0 & 0 & 0.0 & 1 & 1.0 & 0 & 0.0 & 1 & 1.0 & 0 & 0.0 & 1 & 1.0 \\
\hline 1 a 4 años & 8 & 7.8 & 2 & 2.0 & 10 & 9.8 & 3 & 2.9 & 7 & 6.9 & 0 & 0.0 & 10 & 9.8 \\
\hline $\begin{array}{l}\text { Mayor de } 5 \\
\text { años }\end{array}$ & 62 & 60.8 & 29 & 28.4 & 91 & 89.2 & 24 & 23.5 & 65 & 63.7 & 2 & 2.0 & 91 & 89.2 \\
\hline Total & 71 & 69.6 & 31 & 30.4 & 102 & 100.0 & 27 & 26.5 & 73 & 71.6 & 2 & 2.0 & 102 & 100.0 \\
\hline$x^{2}(c)=1.0$ & $x^{2}$ & $=1.38$ & & $\mathrm{Gl}=2$ & & $N . C=0.5$ & & $p<0,0$ & & & & & & \\
\hline$x^{2}(c)=0.67$ & $x^{2}$ & $=3.35$ & & $\mathrm{GI}=4$ & & N.C $=0.5$ & & $\mathrm{p}<0,0$ & & & & & & \\
\hline
\end{tabular}


En el cuadro referente a la satisfacción del personal de salud según tiempo de servicio en la Micro Red de San Juan Bautista, se observa que del total de población en estudio 102 (100\%) el 89.2\% tienen un tiempo de servicio mayor de 5 años, de ellos el $60.8 \%$ tiene satisfacción laboral y $63.7 \%$ tiene un compromiso laboral medio, asimismo el $9.8 \%$ tiene un tiempo de servicio entre 1 a 4 años de ellos $7.8 \%$ tiene satisfacción laboral y $6.9 \%$ tiene un compromiso laboral medio del mismo modo el $1.0 \%$ tiene un tiempo de servicio menor de 1 año y tiene un compromiso laboral medio. De acuerdo a la prueba de chi cuadrada en lo que respecta a la satisfacción se afirma que no existe relación significativa entre satisfacción y tiempo de servicio del personal de salud en la MR de San Juan Bautista. En lo que respecta al compromiso laboral se afirma que no existe relación significativa entre el compromiso laboral y el tiempo de servicio del personal de salud en la MR de SJB.

\section{TABLA No 9}

Compromiso Organizacional según satisfacción laboral del personal de salud de la Micro Red de San Juan Bautista-Ayacucho 2014

\begin{tabular}{cccccccc}
$\begin{array}{c}\text { Compromiso } \\
\text { organizacional }\end{array}$ & \multicolumn{3}{c}{$\begin{array}{c}\text { Satisfacción del personal } \\
\text { Satisfacción }\end{array}$} & \multicolumn{2}{c}{ Insatisfacción } & \multicolumn{2}{c}{ Total } \\
& $\mathbf{N}^{\circ}$ & $\%$ & $\mathbf{N}^{\circ}$ & $\%$ & $\mathbf{N}^{\circ}$ & $\%$ \\
\hline Alta & 26 & 25.5 & 1 & 1.0 & 27 & 26.5 \\
Media & 45 & 44.1 & 28 & 27.5 & 73 & 71.6 \\
Baja & 0 & 0.0 & 2 & 2.0 & 2 & 2.0 \\
\hline Total & 71 & 69.6 & 31 & 30.4 & 102 & 100.0 \\
$\mathrm{X}^{2}$ (c) $=15.9$ & $\mathrm{X}^{2}$ (t) $=1.38$ & $\mathrm{Gl}=2$ & $\mathrm{~N} . \mathrm{C}=0.5$ & $\mathrm{p}>0,05$
\end{tabular}

En el cuadro referente al grado de compromiso organizacional según la satisfacción laboral del personal de salud, se observa que del total de población en estudio 102 (100\%) el 71.6\% tiene compromiso organizacional medio de ellos $44.1 \%$ tiene satisfacción laboral y $27.5 \%$ insatisfacción laboral asimismo $26.5 \%$ tiene compromiso organizacional alto, de ellos $25.5 \%$ tiene satisfacción laboral y $1.0 \%$ insatisfacción laboral, del mismo modo $2.0 \%$ tiene compromiso organizacional bajo e insatisfacción laboral. De acuerdo a la prueba de chi cuadrada se afirma que existe relación significativa entre satisfacción del personal y compromiso laboral.

\section{DISCUSIÓN}

Respecto a la satisfacción del personal de salud en la MR de SJB (cuadro $N^{\circ} 1$ ) Carrasco (34) define la satisfacción laboral como la actitud del trabajador frente a su trabajo. Actitudes determinadas por las características del puesto laboral. García (35) refiere que el personal representa el capital humano de toda organización. Se debe ejecutar estrategias tendientes a ofrecer a los trabajadores elementos que contribuyan a la satisfacción laboral. Se concluye que el mayor porcentaje de trabajadores tiene satisfacción laboral, considerándose que estos resultados son aspectos positivos en el proceso de atención a los usuarios externos, entendiéndose que la satisfacción lleva al mejor desempeño de cada uno de los usuarios internos.

Respecto a la satisfacción según categorías del personal de salud en la MR de San Juan Bautista (cuadro $N^{\circ} 2$ ) Méndez (36) refiere que la satisfacción laboral depende de múltiples factores, de la diversidad de las necesidades de las personas, del contenido de las tareas ejecutadas. Bernat (37) indica que la mayor satisfacción esta dado por la profesionalidad responsable y la naturaleza del trabajo. Se concluye que en general existe satisfacción laboral del personal y las categorías mejor valoradas fueron: el trabajo en general $79.4 \%$ y el ambiente de trabajo con $73.5 \%$ y la menor fue la remuneración e incentivos con $51.0 \%$ y la interacción con el jefe inmediato $51.0 \%$. Respecto al compromiso organizacional del personal de salud en la Micro Red (cuadro $\mathrm{N}^{\circ} 3$ ) Loli (38) en su estudio, muestra que un importante porcentaje de trabajadores tienen una actitud favorable hacia el compromiso organizacional. Robles (33) refiere que el compromiso organizacional es la fuerza de una identificación individual, caracterizado por 3 factores: Fuerte creencia y aceptación de las metas y valores de la organización. Voluntad de hacer esfuerzo a favor de la organización. Deseo de mantener la membrecía organizacional. Se concluye que el mayor porcentaje $71.6 \%$ del personal de salud tiene un compromiso laboral medio.

Respecto a las categorías según compromiso organizacional del personal (cuadro $\mathrm{N}^{\circ} 4$ ) Betanzos (31) indica que el compromiso organizacional tiene dimensiones: Valor del trabajo en sí, compromiso de carrera, involucramiento con el puesto y compromiso con la organización. Se concluye que el mayor porcentaje de trabajadores tiene compromiso organizacional laboral medio (52\%) y las categorías mejor valoradas fueron: identificación (78.4\%) con compromiso medio, lealtad (57.8\%) con compromiso alto. 
Respecto a la satisfacción y compromiso laboral según puesto laboral del personal de salud en la Micro Red de SJB (Cuadro $N^{\circ} 5$ ) Zurita (32) indica respecto al nivel de satisfacción que los trabajadores que tienen mayor nivel de responsabilidad y alto nivel de jerarquía, su nivel de satisfacción va a ir en ascenso. Durrego (39) indica que las teorías señalan que el compromiso tiende a ser mayor cuando las personas tienen altos niveles de responsabilidad. Se concluye que el mayor porcentaje de trabajadores laboran como personal asistencial $71.6 \%$ por considerarse a la Institución como una entidad prestadora de servicios de salud y a su vez el mayor porcentaje de ellos tienen satisfacción y compromiso laboral media, aspecto que se debe mejorar brindando condiciones necesarias para mejorar el compromiso con la Institución.

\section{Respecto a la satisfacción y compromiso laboral según grupo ocupacional (cuadro $N^{\circ} 6$ ) Ángulo (41) señala que la satisfacción de la profesión elegida es un estado afectivo que surge en la persona y determina conductas positivas. Aguilar (1) define la satisfacción como una actitud, está dado por la diferencia entre la calidad de recompensas que recibe y la cantidad que espera recibir. Se concluye que el mayor porcentaje de trabajadores pertenece al grupo ocupacional de Técnicos en Enfermería en un $23.5 \%$ que tiene satisfacción y compromiso laboral medio.}

\section{Respecto a la satisfacción y compromiso laboral según condición laboral del personal de salud de la Micro Red de San Juan Bautista (cuadro $N^{\circ} 7$ ) El Ministerio de Trabajo (42) concluye que la distribución por tipo de contrato se nota que a menor estabilidad laboral, menor satisfacción y a mayor estabilidad mayor es la proporción de trabajadores satisfechos. Se concluye que el mayor porcentaje de población tiene la condición laboral de nombrado (72.5\%) y ellos tienen satisfacción y compromiso laboral medio, aspecto que esta ligado a la actitud de compromiso y otros factores como la condición laboral.}

\footnotetext{
Respecto a la satisfacción y compromiso laboral según tiempo de servicio del personal de salud en la MR de SJB (Cuadro $N^{\circ} 8$ ) Cavalcante (40) menciona que es compleja la relación entre la satisfacción y la antigüedad ya que ésta va cambiando en el transcurso del tiempo. Durrego (39) señala que el número de años que los trabajadores han estado en la organización genera mayor pertenencia y compromiso con la organización. Se concluye que el $89.2 \%$ de los trabajadores cuenta con un tiempo de servicio mayor a 5 años y el mayor porcentaje de ellos tiene satisfacción
}

y compromiso laboral medio, se puede deducir que el tiempo de servicio lleva a un mayor compromiso y la sensación de obligación de permanecer en la Institución.

Respecto al compromiso organizacional según satisfacción laboral (cuadro $\mathrm{N}^{\circ}$ 9) Zurita (32) refiere que existe relación entre la satisfacción y el compromiso laboral por ello es necesario que se respete al empleado. Atalaya (43) afirma que un trabajador contento es un trabajador productivo. Se concluye que el $71.6 \%$ del personal de salud de la MR de SJB tiene un compromiso organizacional medio y de ellos el $44.1 \%$ se encuentra satisfecho, aspecto que nos permite deducir que el empleado que se encuentra satisfecho en su entorno laboral presenta mayor compromiso.

Se concluye que la investigación enfocó la relación existente entre el compromiso y la satisfacción laboral del personal de salud de la MR de SJB encontrándose que el $71.6 \%$ del personal, tienen un compromiso organizacional medio y de ellos el $44.1 \%$ tiene satisfacción personal y según la prueba de chi cuadrado $(p>0,05)$ existe relación significativa entre ambas variables aceptándose la $\mathrm{Hi}$ lo que significa que en la medida que el personal se encuentre más satisfecho tendrá mayor compromiso con el trabajo desempeñado. El mayor porcentaje $69.6 \%$ de trabajadores tiene satisfacción laboral considerándose que éstos resultados son aspectos positivos en el proceso de atención a los usuarios externos. Las categorías mejor valoradas por los trabajadores fueron: identificación $78.4 \%$ con compromiso medio, lealtad $57.8 \%$ con compromiso alto. Según características de la población se encontró que el personal que labora en la Micro Red de SJB trabaja como personal asistencial $71.6 \%$ y tiene una satisfacción y compromiso laboral medio, en relación al grupo ocupacional el $23.5 \%$ trabaja como técnico en enfermería y tiene una satisfacción y compromiso laboral medio, respecto a la condición laboral el $72.5 \%$ es nombrado y tiene satisfacción y compromiso laboral medio, en lo que respecta al tiempo de servicio el $89.2 \%$ de trabajadores cuenta con un tiempo de servicio mayor de 5 años y tiene un nivel de satisfacción y compromiso laboral medio.

Se recomienda que los prestadores de servicios de salud se encuentran algo satisfechos es recomendable que el Ministerio de Salud implemente estrategias que refuercen la formación de valores intrínsecos como es el respeto, confianza, identidad con la Institución que genere una fuerza de trabajo con altos niveles de 
satisfacción y compromiso laboral. A la DIRESA y Micro Redes se le recomienda realizar capacitaciones frecuentes, sensibilización, para involucrar más al personal y hacerlos partícipes de los procesos y Plan Operativo Institucional.

\section{REFERENCIAS BIBLIOGRÁFICAS}

1. Aguilar Morales Norma. Importancia de la satisfacción laboral [Tesis maestría]. México: Universidad Juárez Autónoma de Tabasco; Escuela de Post Grado, 2010. Disponible en

http://www.itson.mx/publicaciones/pacioli/Documents /69/38-importancia_de_la_satisfacción_laboral _investigación_octubre_2010x.p.pdf

2. Bonadeo Miriam A, et al. Evaluación del Grado de Satisfacción del Personal Médico en un Hospital de Pediatría. Arch.Argent. pediatr. [online].2005. Vol.103, n.6, pp491-496.ISSN 1668-3501. Disponible en: http//www.scielo.org.ar/scielo.php?pid=arttext

3. Bobbio Lucía. Satisfacción Laboral y factores asociados en Personal Asistencial Médico y no Médico de un Hospital Nacional de Lima-Perú. Rev.Perú. Epidemiol. Vol. 14 №2 Agosto 2010.pp.133-138, disponible en:

http://www.redalyc.org/artículo.oa?id=203119666007.

4. Pavón León Patricia. Satisfacción del Personal de Salud en un Hospital Universitario de Salud en Tabasco. Vol. 17, $\mathrm{N}^{\circ} 1$ y 2. Enero-Abril, Mayo-Agosto 2011, pp. 13-21. México. http://www.redalyc.org/pdf/487/48721182003.pdf

5. Chiang V. Margarita. Compromiso del Trabajador hacia su Organización y la Relación con el Clima Organizacional: Un análisis de Género y Edad. Panorama Socioeconómico Año 28, $N^{\circ} 40$, pp92-103 Julio 2010. http://www.panorama.utalca.cl/dentro/2010jul/artículo7.pdf

6. Llapa Rodríguez EO, Trevizan M.A, Shinyashiki T.G, Méndez Costa IA. Compromiso Organizacional y Profesional del Equipo de Salud. Enferm. Glob. [revista en la internet]. 2009 Oct. [citado 2014 Abr. 01]; (17): Disponible en:

http://scielo.isciii.es/scielo.php?script=sci_arttext\&pid= S1695-61412009000300013\&Ing=es.

7. Alonso Martín Pilar. Estudio Comparativo de la Satisfacción Laboral en el Personal de Administración. Revista de Psicología del Trabajo y de las Organizaciones [en línea]2008. Vol. 24 [citado 2012-08.10]. Disponible en: http://redalyc.uaemex.mx/src/inicio/ ArtpdfRed.jsp?¡Cve=231316494002. ISSN 1576-5962.

8. García Molto A. Satisfacción Laboral y Diferencias Motivacionales y de Personalidad en Lesiones Medulares con distinta situación de empleo. [Tesis Doctoral]. Madrid: Editorial de la Universidad Complutense de Madrid; Facultad de Psicología, 2003.

9. Jauli Dávila I. Las actitudes ante los Errores en los Mandos Intermedios de una Organización. [Tesis Doctoral]. Madrid: Editorial de la Universidad Complutense de Madrid: Facultad de Psicología. 1997.

10. Subirats Ferrer M. Clima Perceptivo y Satisfacción Laboral en Equipos de Trabajo: Un análisis longitudinal y multinivel. [Tesis Doctoral]. Universidad de Valencia.
Facultad de Psicología. 2000.

11. Ponce Gómez J, Ponce Gómez G. Satisfacción Laboral del Personal de Enfermería en un Hospital de Gineco Obstetricia del Instituto Mexicano del Seguro Social. Red de Investigación sobre factores psicosociales en el Trabajo. [serie en internet] 2009 [consultado 17 Febrero 2012]. Disponible

en:http://factorespsicosociales.com/artículos/?autor=4 \&paged $=2$

12. Gonzales Huertas D, Loconi Moreno C, Sánchez Salas V, Ochoa Vigo K. Determinantes de la Satisfacción en el Trabajo de las Enfermeras Comunitarias de la DISA V, Lima Ciudad. Revenferm Herediana. 2008; 1(2):71-79.

13. Fernández S. Clima Laboral de los Trabajadores de la Municipalidad José Leonardo Ortiz. Chiclayo-Perú. 2006.

14. Alfaro Salazar Ronald. Satisfacción Laboral y su Relación con algunas variables Ocupacionales en Tres Municipalidades [Tesis Maestría]. Lima Perú: Pontificia Ubiversidad Católica del Perú; Escuela de Posgrado. Julio de 2012. Disponible en:

http//tesis.pucp.edu.pe/repositorio/bitstream/handle/ 123456789/4593/ALFARO_LEYTON_MEZA_SAENZ_SATI SFACCION_LABORAL.pdf?sequence $=1$

15. Robbins S.P. Comportamiento Organizacional. México 8va. Edición D.F. Prentice-Hall-Hispanoamérica, S.A. 1987.

16. Abrajan Castro María Guadalupe, Contreras Padilla José Manuel. Grado de Satisfacción Laboral y Condiciones de Trabajo: Una exploración cualitativa. Enseñanza e investigación en Psicología. Vol. 14, núm. 1, enero-junio, 2009, pp. 105-118. Consejo Nacional para la Enseñnz en Investigación en Psicología. México. Disponible en: http://www.redalyc.org/pdf/292729214108.pdf

17. Gargallo Castel Ana. La Satisfacción Laboral y sus determinantes en las Cooperativas. Enseñamza e Investigación en Psicología.Vol.12, núm. 1, Setiembre, 2010, pp.563-575. México

http://www.google.com.pe/url?sa $=t \& r c t=j \& q=\& e s r c=s$ $\&$ frm $=1 \&$ source $=$ web \&cd $=7 \&$ cad $=$ rja $\&$ uact $=8 \&$ ved $=0 C$ EsQFjAG\&url=htt'\%3A\%2F\%2Fdialnet.unirioja.es\%2Fdes carga\%2Fartículo\%2F2774951.pdf\&ei=_AolVbCXEsS5gg SN80GYDg\&usg=AFQjCNFxOKnvnzTB4xQAfRuEDr_PboZ 6Vw6sig2=VzbWD8suwHj6dOQVf9QVf9QH5A

18. Loitegui A.JR. Determinantes de la Satisfacción Laboral en Empleados de la Administración Foral de Navarra. [Tesis Doctoral]. Universidad Complutense de Madrid: Facultad de Psicología. 1990.

19. Kreitner, Kinicki A. Comportamiento de las Organizaciones. Madrid 1997.

20. Gonzales Tirados RM. Los Recursos Humanos en las Universidades y su relación con la calidad de la enseñanza. En actas I Congreso Internacional sobre 
calidad de la enseñanza Universitaria. Universidad de Cádiz. ICE, PPP, 184-1911991.

21. Sáenz Barrio O, Lorenzo Delgado, M. (Eds). La satisfacción del profesorado Universitario. Monografía. Pedagogía. Universidad de Granada 1993.

22. Porter LW, Steers RM, Mowday RT, Boulian PV. Organizational commitment, job satisfaction, and turnover, among psychiatric technicians. [Journal of applied Psychology, 59-60[609] 1974.

23. Mathieu JE, Zajac DM. Review and Meta-analysis of the Antecedents, Correlates and Consequences of Organizational Commitment. 1990; 180, pp 171-194.

24. MINSA. Encuesta de Satisfacción del Personal de Salud. Dirección General de Salud de las Personas. Lima-Perú, 2002.

25. Meyer JP, Allen J. Tres Componentes, Conceptualización de Compromiso Organizacional. Human Resource Management Review 2001,1(1): 61-98.

26. Díaz Bretones F, Montalbán Peregrín M. El individuo como unidad de Análisis: La Influencia de los Valores, Percepciones y Actitudes en el Comportamiento Laboral. Psicología de las Organizaciones. Barcelona. Editorial UOC 2004.

27. Jiménez Jl. El Compromiso Laboral. Diario el País [en internet\} Oct. 2007 [consulado 20 Mayo 2012]. Disponible en.

http://elpais.com/diario/2007/10/07/negocio/1191762 867850215.html

28. Rivera Ramón L. Compromiso Laboral. Puerto Rico Julio 2009.

29. Rivera Carrascal Oscar Martín. Compromiso Organizacional de los Docentes de una Institución Educativa Privada de Lima Metropolitana y su correlación con variables Demográficas. Julio 2010. 25(98) Tesis UPCP-Lima

http://tesis.pucp.edu.pe/repositorio/bitstream/handle/ 123456789/48077RIVERA_CARRASCAL_OSCAR_COMPR OMISO_DEMOGRÁFICAS.p̄ff?sequence $=1$

30. Teresa Vila María. Relación entre Compromiso Organizacional y Satisfacción Laboral en una Empresa del
Sector Financiero. Univ. Católica Andrés Bello- Caracas 2005. Pp 68-84.

http://biblioteca2.ucab.edu.ve/anexos/biblioteca/marc/ texto/AAP1795.pdf

31. Betanzos Días Norma, et al. Compromiso Organizacional en Profesionales de la Salud. Revisión bibliográfica Universidad Autónomas del Estado de More. Rev. Enferm Inst. Méx. Seguro Soc. 2011,19 (19:35-41. http//www.medigraphic.com/pdfs7enfermeríaimss7ei m-20117eim111h.pdf

32. Zurita Alcaraz Marta Carmen. Compromiso Organizacional y Satisfacción Laboral en una Muestra de Trabajadores de los Jusgados de Granada. ReiDoCreavolumen 3 artículo 3 p 17-25 Abril 2014. http://digibug.ugr.es7bitstream/10481/31291/1/ReiDo Crea-Vol.3-Zurita-Ramirez-Quezada-Quezada-RuizManzano.pdf

33. Robles Hernández José Antonio. El Compromiso Organizacional y la Actitud de los Profesores de Ciencias económicas Administrativas de la Inst. Públicas frente a las necesidades de Capacitación Institucional. Facultad de Contaduría y Administración Rev.

CIENCIA@UAQ.2(2):2009.

http://www.uaq.mx/investigación/revista_ciencia@uaq /ArchivosPDF/v2-n2/Compromiso.pdf

34. Carrasco Soto Raymundo, Barraza Masías, Arturo, et al. Satisfacción Laboral en Personal del Centro de Salud N¹ de la Ciudad de Durango, México. (2013,23 de Agosto). Psico Pedia hoy, 15(2). Disponible en:

http://psicopediahoy.com/satisfaccion-laboral-centrode-salud

35. García Ramos Martina, Luján López María Elena. Satisfacción Laboral del Personal de Salud. Revista de Enfermería Instituto México Seguro Social 2007, pp. 6372 http://www.medigraphic.com/pdfs/enfermeriaimss/eim-2007/eim072b.pdf

\section{Correspondencia}

Nelly Felipa Ledesma Zerpa

nellyydiego@hotmail.com
Fecha de recepción: 05 de agosto de 2017

Fecha de aceptación: 05 de octubre de 2017 